\title{
Hypothalamic injury in spontaneous subarachnoid hemorrhage: a diffusion tensor imaging study
}

\author{
Sung Jun Lee ${ }^{1}$. Sung Ho Jang ${ }^{1}$
}

Received: 2 September 2020 / Accepted: 6 November 2020 / Published online: 28 November 2020

(c) The Author(s) 2020

Keywords Hypothalamus · Subarachnoid hemorrhage · Diffusion tensor imaging · Fractional anisotropy $\cdot$ Apparent diffusion coefficient

Dear Editors,

Spontaneous subarachnoid hemorrhage (SAH), which accounts for $3 \%$ to $5 \%$ of all strokes, is mainly the result of the extravasation of blood into the subarachnoid space, caused by aneurysmal rupture of the cerebral artery [1]. It has been reported that more than half of patients with SAH show neurologic manifestations related to brain injury [1]. Several pathophysiologic mechanisms involved in SAH-related brain injury have been suggested, including global vasogenic edema, vasospasm and cerebral ischemia, mechanical injury (via increased intracranial pressure (barotrauma from pressure waves) or a direct mass effect of the $\mathrm{SAH}$ ), and chemical injury (a blood clot can cause neural injury through the release of potentially damaging substances, such as free iron, which may result in the generation of free radicals or inflammatory cytokines) [2]. Through these SAH-related mechanisms, a SAH can affect extensive brain areas, including specific neural structures such as the cingulum, fornix, and mamillothalamic tract [3].

Various clinical manifestations related to autonomic dysfunction in SAH have been reported, including hyperthermia, hypertension, cardiac dysrhythmia, impaired consciousness, and hyperhidrosis [4]. Among the sequelae of SAH, paroxysmal

Electronic supplementary material The online version of this article (https://doi.org/10.1007/s10286-020-00747-5) contains supplementary material, which is available to authorized users.

Sung Ho Jang

strokerehab@hanmail.net

Sung Jun Lee

hssj8020@hanmail.net

1 Department of Physical Medicine and Rehabilitation, College of Medicine, Yeungnam University, 317-1, Daemyungdong, Namku, Taegu 705-717, Republic of Korea sympathetic hyperactivity, which is caused by dysfunction of portions of the central autonomic network (including the hypothalamus, amygdala, insular cortex, and brainstem), is a representative SAH-related autonomic disease [5]. The incidence of paroxysmal sympathetic hyperactivity of patients with SAH has been reported to be approximately $11.3 \%$.

The hypothalamus, a central mediator of the autonomic nervous system, has essential roles in the autonomic functions of the human body (e.g., thermoregulation, regulation of food intake and body water content, endocrine control, reproduction, and circadian rhythm) [6]. Clinical manifestations caused by autonomic system dysfunction due to the hypothalamic injury are disturbance of thermoregulation, impaired consciousness, and hyperhidrosis, and these manifestations overlap with those of SAH [6]. Because the hypothalamus is anatomically located adjacent to the subarachnoid space and large subarachnoid cisterns, including the interpeduncular and chiasmatic cisterns, the hypothalamus could be vulnerable to SAH (supplemental fig. 1-A). However, precise estimation of the hypothalamus in the live human brain has been limited because of the anatomical characteristics of the hypothalamus, which is very small and only occupies approximately $0.5 \%$ of the whole brain volume; moreover, it has a deep location within brain white matter [7]. Regardless, diffusion tensor imaging (DTI) has enabled evaluation of the hypothalamus in the live human brain. Several studies using DTI have reported on hypothalamic injuries in patients with various brain diseases, including traumatic brain injury, hypoxic-ischemic brain injury, and multiple sclerosis [8-10]. However, no DTI study on the hypothalamic injury in patients with SAH has been reported.

In this study, we hypothesized that the hypothalamic injury could occur in the patients with SAH and investigated it using DTI. Detailed methods, demographic data, and clinical information are provided in supplemental file 1 . 
Table 1 Comparison of the diffusion tensor imaging parameters between the patient and control groups

\begin{tabular}{lll}
\hline & FA & ADC \\
\hline Patient group & $0.23 \pm 0.03$ & $1.54 \pm 0.23$ \\
Control group & $0.26 \pm 0.03$ & $1.02 \pm 0.13$ \\
$p$ value & $0.001^{*}$ & $0.001^{*}$ \\
\hline
\end{tabular}

Values represent mean ( \pm standard deviation)

$F A$ fractional anisotropy, $A D C$ apparent diffusion coefficient

*Significant differences between the patient and control groups, $p<0.05$

The results of the comparison of the DTI parameters of the patient and control groups are summarized in Table 1. The mean fractional anisotropy (FA) value was significantly lower in the patient group than in the control group $(p<0.05)$, whereas the mean apparent diffusion coefficient (ADC) value was significantly higher in the patient group than in the control group $(p<0.05)$.

The results of correlations between clinical information and the DTI parameters are provided in supplemental file 1.

In the current study, by using DTI, the hypothalamus was estimated in control subjects and patients with spontaneous $\mathrm{SAH}$. The FA value was lower, and the ADC value higher, in the patient group than in the control group. Regarding DTI parameters, FA, which is a scalar measure, represents the level of white matter organization and is influenced by axonal myelination, density, and the degree of directionality and level of organization of white matter tracks. A decrement in the FA value indicates impaired integrity of the white matter. The ADC value is indicative of the magnitude of water diffusion in brain tissue, which can increase with some disease forms, particularly vasogenic edema, or through the accumulation of cellular debris from neuronal injury. Therefore, the statistically significant decrement in FA value and increment in ADC value appear to indicate the presence of injuries of the hypothalamus in the SAH patients. We think that the hypothalamic injury might be related with the blood in the subarachnoid space and large subarachnoid cisterns including the interpeduncular and chiasmatic cisterns adjacent to the hypothalamus. However, we were unable to discern whether the injuries were associated with mechanical or chemical factors. Therefore, further studies on this topic are warranted. Additional discussion is provided in supplemental file 1 .

In conclusion, by using DTI, we demonstrated hypothalamic injuries in patients with spontaneous SAH. The results of this study suggest that our methodology could be helpful in research on the hypothalamus of patients with SAH. Accurate DTI-based estimation of the hypothalamus is deemed necessary in SAH patients showing clinical manifestations of autonomic dysfunction.
Acknowledgements This work was supported by the Medical Research Center Program (2015R1A5A2009124) through the National Research Foundation of Korea (NRF) funded by the Ministry of Science, ICT and Future Planning.

Funding Financial disclosure statements have been obtained, and no conflicts of interest have been reported by the authors or by any individuals in control of the content of this article.

\section{Compliance with ethical standards}

Conflicts of interest The authors declare that they have no competing interests.

Open Access This article is licensed under a Creative Commons Attribution 4.0 International License, which permits use, sharing, adaptation, distribution and reproduction in any medium or format, as long as you give appropriate credit to the original author(s) and the source, provide a link to the Creative Commons licence, and indicate if changes were made. The images or other third party material in this article are included in the article's Creative Commons licence, unless indicated otherwise in a credit line to the material. If material is not included in the article's Creative Commons licence and your intended use is not permitted by statutory regulation or exceeds the permitted use, you will need to obtain permission directly from the copyright holder. To view a copy of this licence, visit http://creativecommons.org/licenses/by/4.0/.

\section{References}

1. van Gijn J, Kerr RS, Rinkel GJ (2007) Subarachnoid haemorrhage. Lancet 369:306-318

2. Lee SJ, Kim MS, Jang SH (2020) White matter abnormalities in spontaneous subarachnoid hemorrhage: a tract-based spatial statistics study. Stroke 51(9):e246-e249

3. Cho MK, Jang SH (2020) Diffusion tensor imaging studies on spontaneous subarachnoid hemorrhage-related brain injury: a mini-review. Front Neurol 11:283

4. Harland TA, Seinfeld J, Cava LF, Neumann RT, Roark C, Kumpe D et al (2019) Anti-neutrophil cytoplasmic antibody associated central nervous system vasculitis with brain and spinal cord subarachnoid hemorrhage: a rare case report and review of the literature. J Clin Neurosci 62:253-255

5. Benarroch EE (1993) The central autonomic network: functional organization, dysfunction, and perspective. Mayo Clin Proc 68:988-1001

6. Pop MG, Crivii G, Opincariu I (2018) Anatomy and function of the hypothalamus. Intech open, London

7. Baroncini M, Jissendi P, Balland E, Besson P, Pruvo JP, Francke JP et al (2012) MRI atlas of the human hypothalamus. Neuroimage 59:168-180

8. Shen Y, Bai L, Gao Y, Cui F, Tan Z, Tao Y et al (2014) Depressive symptoms in multiple sclerosis from an in vivo study with TBSS. Biomed Res Int 2014:148465

9. Jang SH, Yi JH, Kim SH, Kwon HG (2016) Relation between injury of the hypothalamus and subjective excessive daytime sleepiness in patients with mild traumatic brain injury. J Neurol Neurosurg Psychiatry 87:1260-1261

10. Jang SH, Kwon HG (2018) Injury of the hypothalamus in patients with hypoxic-ischemic brain injury. Am J Phys Med Rehabil 97:160-163 